\title{
Comparação do nível de dor femoropatelar, atividade física e qualidade de vida entre adolescentes do sexo feminino e masculino
}

\author{
Comparison of the level of patellofemoral pain, physical activity, and quality \\ of life between female and male adolescents
}

\author{
Marina Cabral Waiteman', Maira Bergamaschi Coura', Cynthia Gobbi Alves Araújo², Ronaldo Valdir Briani², \\ Danilo de Oliveira Silva ${ }^{2} \bowtie$, Fábio Mícolis de Azevedo ${ }^{2,3}$ \\ ${ }^{1}$ Discente do Curso de Graduação em Fisioterapia da Universidade Estadual Paulista Júlio de Mesquita Filho (UNESP). Presidente Prudente, SP \\ 2 Programa de Pós-Graduação em Fisioterapia da UNESP. Presidente Prudente, SP. \\ ${ }^{3}$ Professor Associado do Departamento de Fisioterapia da UNESP. Presidente Prudente, SP.
}

\section{RESUMO}

Objetivos: Comparar o nível da dor, o nível de atividade física e a qualidade de vida de adolescentes do sexo feminino e masculino com dor femoropatelar.

Métodos: Foram recrutados de escolas públicas e privadas da cidade de Presidente Prudente, São Paulo, 46 adolescentes do sexo feminino e 46 adolescentes do sexo masculino entre 12 e 18 anos. Para serem incluídos no estudo os adolescentes precisavam referir dor no joelho de início insidioso, exacerbada em algumas atividades apresentadas e com no mínimo seis semanas de duração. Além disso, os adolescentes deveriam reportar pior dor ao longo da semana anterior de pelo menos $30 \mathrm{~mm}$ na Escala Visual Analógica e não poderiam apresentar sinais, sintomas ou alguma ocorrência de qualquer outra disfunção no joelho. Os participantes foram solicitados a marcarem na Escala Visual Analógica o nível de dor no joelho auto reportado do último mês. A avaliação do nível de atividade física foi realizada por meio da aplicação do Baecke Questionnaire of Habitual Physical Activity e para investigar a qualidade de vida dos participantes, a subescala de qualidade de vida do questionário Knee Outcome in Osteoarthritis Survey foi utilizada. O teste t-independente foi utilizado para identificar diferenças entre os grupos em relação ao nível de atividade física, qualidade de vida e dor no joelho.

Resultados: Pela Escala Visual Analógica os adolescentes do sexo masculino apresentaram nível de dor no joelho no mês anterior de 5,1 $\pm 1,4$ e as adolescentes do sexo feminino de $4,4 \pm 1,6(\mathrm{p}=0,029)$. O escore de atividade física foi de $8,8 \pm 1,3$ para os adolescentes do sexo masculino e de $7,5 \pm 1,6$ para as do sexo feminino $(\mathrm{p}<0,001)$. A subescala de qualidade de vida resultou em $77,9 \pm 19,0$ para as meninas adolescentes e em $70,8 \pm 13,5$ para os adolescentes do sexo masculino $(\mathrm{p}=0,042)$.

Conclusões: Entre adolescentes com dor femoropatelar, os do sexo masculino apresentaram maior nível de dor no joelho, maior nível de atividade física e menor qualidade de vida em comparação com as adolescentes do sexo feminino.

DESCRITORES: síndrome da dor femoropatelar; adolescente; qualidade de vida.

\section{ABSTRACT}

Aims: To compare the level of pain, physical activity, and quality of life of female and male adolescents with patellofemoral pain.

Methods: A total of 46 female adolescents and 46 male adolescents aged 12 to 18 years were recruited from public and private schools in the town of Presidente Prudente, São Paulo, Brazil. To be included in the study, adolescents needed to report knee pain of insidious onset, exacerbated in some activities, and lasting for at least 6 weeks. In addition, adolescents should report worse pain over the previous week of at least $30 \mathrm{~mm}$ on the Visual Analog Scale and could not show signs, symptoms or any occurrence of any other knee dysfunction. The participants were requested to mark their self-reported knee pain in the last month on a Visual Analog Scale. The level of physical activity was evaluated by the Baecke Questionnaire of Habitual Physical Activity and the quality of life subscale of the Knee Outcome in Osteoarthritis Survey questionnaire was used to investigate the participants' quality of life. The independent t-test was used to find differences between the groups regarding the level of physical activity, quality of life, and knee pain.

Results: According to the Visual Analog Scale, male adolescents presented a level of knee pain of $5.1 \pm 1.4$ in the previous month and female adolescents, of $4.4 \pm 1.6(\mathrm{p}=0.029)$. The physical activity score was $8.8 \pm 1.3$ for male adolescents and $7.5 \pm 1.6$ for female ones $(\mathrm{p}<0.001)$. The quality of life subscale scores were $77.9 \pm 19.0$ for adolescent girls and $70.8 \pm 13.5$ for male adolescents $(\mathrm{p}=0.042)$.

Conclusions: Among adolescents with patellofemoral pain, males had a higher level of knee pain, higher level of physical activity, and lower quality of life compared to female adolescents.

KEY WORDS: patellofemoral pain syndrome; adolescent; quality of life. 
Abreviaturas: DFP, dor femoropatelar; FCT/UNESP, Faculdade de Ciências e Tecnologia, Universidade Estadual Paulista Júlio de Mesquita Filho; KOOS, Knee Outcome in Osteoarthritis Survey; EVA, Escala Visual Analógica; BQHPA, Baecke Questionnaire of Habitual Physical Activity.

\section{INTRODUÇÃO}

A dor femoropatelar (DFP) é um distúrbio caracterizado por dor anterior, retro ou peripatelar, agravada por atividades que sobrecarregam a articulação femoropatelar, como agachamento, subida e descida de escadas, corrida e saltos [1,2]. Além disso, é uma condição que, na maioria das vezes, está presente na população fisicamente ativa e é responsável por impactar não somente a participação em esportes, mas também a qualidade de vida e as atividades de vida diária $[3,4]$. Esse distúrbio musculoesquelético é comum entre adolescentes, acometendo cerca de 6 a 7\% dessa população [5]. Em adolescentes praticantes de esportes, como o basquete, pode acometer até $16 \%$ [6]. Em um estudo realizado com indivíduos com DFP entre 20 e 30 anos de idade, $57 \%$ da população obtiveram prognóstico desfavorável com recidiva dos sintomas em um período de 5 a 8 anos após o tratamento [7]. Entretanto, apesar do prognóstico desfavorável, sua etiologia permanece desconhecida [7-9].

Apesar da alta incidência nessa população, a DFP em adolescentes é frequentemente negligenciada. De acordo com Rathleff et al. [10], mais de 1500 artigos científicos já foram publicados na área da DFP nos últimos 30 anos e menos de $5 \%$ desses estudos envolvem adolescentes. Na maioria dos casos, adolescentes com DFP apresentam sintomatologia de mais de três anos [11], e apenas 5\% reportam duração da dor por menos de seis meses [12]. Além da dor a longo prazo, adolescentes com DFP reportam reduções no nível funcional e na qualidade de vida [13]. Sabe-se, também, que adolescentes praticam altos níveis de atividade física e esse fator está associado com a persistência da dor [14]. De Oliveira Silva et al. [3] reportaram que a gravidade dos sintomas da DFP associa-se com a redução na qualidade de vida de atletas adolescentes com DFP. De maneira semelhante, Cheung et al. [15] mostraram que atletas de alto rendimento com DFP apresentaram menores índices de qualidade de vida em comparação com atletas amadores com DFP. Portanto, parece que o nível de atividade física dos adolescentes pode influenciar a sua qualidade de vida.
Adolescentes do sexo feminino tem 2,3 vezes mais chance de desenvolver DFP em comparação a adolescentes do sexo masculino [16]. Além disso, as meninas adolescentes são caracterizadas por maiores alterações no controle motor e incapacidade de absorção de cargas, o que favorece menor estabilização patelar em comparação ao sexo masculino $[17,18]$. Embora alguns estudos tenham investigado a qualidade de vida, o nível de atividade física e a dor de adolescentes com DFP [3,13,15], ao nosso conhecimento nenhum estudo investigou essas características considerando o sexo. Assim, este estudo teve por objetivo comparar o nível da dor no joelho, o nível de atividade física e a qualidade de vida de adolescentes do sexo feminino e masculino com DFP.

\section{MÉTODOS}

Este estudo transversal incluiu adolescentes recrutados de escolas públicas e privadas da cidade de Presidente Prudente, São Paulo. O tamanho amostral foi baseado nos dados de De Oliveira Silva et al. [3] para um teste com poder preditivo de $80 \%$ e $\alpha=0,05$. Considerando uma diferença esperada entre os grupos de 10 pontos no escore do domínio qualidade de vida do questionário Knee Outcome in Osteoarthritis Survey (KOOS) e um desvio padrão de 18,77 pontos, o tamanho mínimo da amostra indicado foi de 43 adolescentes por grupo.

Os critérios de inclusão dos adolescentes foram baseados em estudos prévios [3,13,19]: (1) possuir idade entre 12 e 18 anos; (2) referir dor anterior ou retropatelar de início insidioso com no mínimo seis semanas de duração; (3) reportar dor em no mínimo duas das seguintes atividades: agachamento, subir e/ou descer escadas, permanecer sentado por tempo prolongado, corrida, prática de esportes e saltos/ aterrisagens; (4) reportar pior dor ao longo da semana anterior de pelo menos $30 \mathrm{~mm}$ na Escala Visual Analógica (EVA) de 100 milímetros. Era necessário que os participantes apresentassem todos os critérios para serem considerados com DFP.

Os critérios de não inclusão foram sinais ou sintomas de qualquer outra disfunção no joelho, história recente (dentro de seis meses) de cirurgia nessa articulação, história de subluxação patelar ou uma evidência clínica de lesão meniscal, instabilidade ligamentar, osteoartrose, patologia no tendão patelar, ou dor referida vinda da coluna vertebral; presença de doença neurológica; presença de processo inflamatório ou sintomas de sobrecarga; fisioterapia prévia (pelo menos seis meses). 
Todos os adolescentes foram avaliados por dois fisioterapeutas com cinco anos de prática clínica e foram incluídos no estudo somente se os dois fisioterapeutas entrassem em acordo sobre a condição do adolescente. No caso de discordância, um terceiro fisioterapeuta conduzia uma avaliação.

A EVA foi utilizada para definir a dor autorreportada no último mês. Essa escala consiste de uma linha de $100 \mathrm{~mm}$, onde 0 significa nenhuma dor e 100 significa a pior dor possível. Os participantes foram solicitados a marcar na linha a intensidade média da dor sentida no mês anterior, a qual foi posteriormente convertida em dados numéricos [20]. Essa escala é validada e reprodutível para a avaliação de indivíduos com DFP [20].

A avaliação do nível de atividade física foi realizada por meio da aplicação de um questionário comumente utilizado para avaliar adolescentes, o Baecke Questionnaire of Habitual Physical Activity (BQHPA). O BQHPA é composto por 16 questões distribuídas em três dimensões que abrangem atividades físicas na escola ou no trabalho; nos esportes e práticas de lazer ativo; e na ocupação do tempo livre e locomoção. As respostas obtidas em cada dimensão foram codificadas em escalas de cinco pontos (1) nunca, (2) raramente, (3) algumas vezes, (4) frequentemente e (5) sempre [21]. Por meio da soma das dimensões é possível estimar o nível de atividade física habitual de adolescentes.

Para investigar a qualidade de vida dos participantes, o domínio qualidade de vida do questionário KOOS foi utilizada. O KOOS é um questionário de 42 itens, que abrange cinco domínios: dor, sintomas, atividades de vida diária, esporte e função recreativa e qualidade de vida relacionado ao joelho. Cada dimensão tem a sua própria pontuação que varia de 0 a 100 . A dimensão de interesse deste estudo foi a qualidade de vida relacionada ao joelho, com maior pontuação indicando melhor qualidade de vida e pontuações mais baixas indicando pior qualidade de vida [22].

Um avaliador foi responsável pela aplicação dos questionários nos participantes. Não foi permitido que mais de três voluntários respondessem aos questionários simultaneamente, dessa forma o avaliador foi capaz de fornecer qualquer assistência necessária. A aplicação dos questionários foi realizada nas próprias escolas, no período de agosto a dezembro de 2015.

Os responsáveis legais juridicamente pelos voluntários que participaram da pesquisa assinaram ao termo de consentimento livre e esclarecido para participação na pesquisa e os adolescentes assinaram o termo de assentimento, elaborado conforme resolução 466/12 do Conselho Nacional de Saúde. O projeto foi aprovado pelo Comitê de Ética em Pesquisa da Universidade Estadual Paulista Júlio de Mesquita Filho sob parecer consubstanciado de número 1.484.129.

Os dados foram tabulados em planilhas prédefinidas no Microsoft Office Excel para Windows. Todas as análises foram conduzidas no programa IBM SPSS 18.0. Foi realizada análise descritiva dos dados em média e desvio padrão. Os dados foram testados quanto à distribuição normal pelo teste Shapiro-Wilk. $\mathrm{O}$ teste t-independente foi utilizado para identificar diferenças entre os grupos de adolescentes (sexo feminino e masculino) em relação ao nível de atividade física, qualidade de vida e dor no joelho. Para todas as análises foi considerado nível de significância de $\alpha<0,05$.

\section{RESULTADOS}

A amostra do estudo foi composta por 92 adolescentes (faixa etária de 12 a 18 anos conforme critério de inclusão) com DFP, sendo 46 do sexo masculino e 46 do sexo feminino. Os dados antropométricos, nível de dor no joelho, atividade de física e qualidade de vida dos participantes estão descritos na Tabela 1. Não houve diferença entre grupos em relação aos dados antropométricos.

Os adolescentes do sexo masculino apresentaram maiores níveis de dor no joelho em comparação com as adolescentes do sexo feminino $\left(\mathrm{t}_{(90)}=-2.214, \mathrm{p}=0,029\right)$. Além disso, os adolescentes do sexo masculino apresentaram maiores níveis de atividade física do que adolescentes do sexo feminino $\left(\mathrm{t}_{(90)}=-3.997, \mathrm{p}<0,001\right)$. Os adolescentes do sexo masculino apresentaram menores escores no domínio qualidade de vida do KOOS quando comparados as adolescentes do sexo feminino $\left(\mathrm{t}_{(90)}=2.060, \mathrm{p}=0,042\right)$ (Tabela 1).

Tabela 1. Dados antropométricos, dor, atividade física e qualidade de vida de adolescentes com dor femoropatelar, conforme o sexo. Presidente Prudente, São Paulo, 2015.

\begin{tabular}{lccc}
\hline \multicolumn{1}{c}{ Variáveis } & $\begin{array}{c}\text { Sexo feminino } \\
\text { (Média } \pm \text { DP) }\end{array}$ & $\begin{array}{c}\text { Sexo masculino } \\
\text { (Média } \pm \text { DP) }\end{array}$ & $\mathbf{p}^{*}$ \\
\hline Idade (anos) & $14,6 \pm 1,5$ & $14,9 \pm 1,7$ & 0,390 \\
Peso $(\mathrm{kg})$ & $62,5 \pm 48,3$ & $78,0 \pm 90,8$ & 0,311 \\
Altura $(\mathrm{m})$ & $1,43 \pm 0,50$ & $1,33 \pm 0,67$ & 0,416 \\
Dor do mês anterior (EVA) & $4,4 \pm 1,6$ & $5,1 \pm 1,4$ & $\mathbf{0 , 0 2 9}$ \\
BQHPA escore & $7,5 \pm 1,6$ & $8,8 \pm 1,3$ & $\mathbf{0 , 0 0 1}$ \\
KOOS-QV escore & $77,9 \pm 19,0$ & $70,8 \pm 13,5$ & $\mathbf{0 , 0 4 2}$ \\
\hline
\end{tabular}

DP, desvio padrão; EVA, Escala Visual Analógica; KOOS-QV, domínio qualidade de vida do Knee Outcome in Osteoarthritis Survey; BQHPA, Baecke Questionnaire of Habitual Physical Activity.

* Teste $\mathrm{t}$ independente. 


\section{DISCUSSÃO}

Contrariamente ao esperado, identificou-se que adolescentes do sexo masculino com DFP apresentaram maiores níveis de dor no joelho bem como maiores níveis de atividade física do que adolescentes do sexo feminino. Além disso, adolescentes do sexo masculino reportaram menor qualidade de vida quando comparados às adolescentes do sexo feminino.

Embora a prevalência da DFP seja maior em mulheres e em adolescentes do sexo feminino $[10,23]$, o presente estudo mostra que adolescentes do sexo masculino com DFP possuíam maiores níveis de dor autorreportada. Isso pode indicar que, embora as adolescentes do sexo feminino sejam mais acometidas pela DFP, os adolescentes do sexo masculino apresentam maior severidade dos sintomas.

O nível elevado de dor no joelho dos adolescentes com DFP do sexo masculino em comparação ao sexo feminino pode estar relacionado ao maior nível de atividade física dessa população. Em estudo de Briani et al. [4], indivíduos com DFP que apresentavam maiores níveis de atividade física reportaram maior dor no joelho em comparação com indivíduos que praticavam menores níveis de atividade física. Além disso, aquele estudo reportou que indivíduos que praticavam altos níveis de atividade física apresentaram atraso no início da ativação do músculo vasto medial em relação ao vasto lateral durante subida e descida de escada quando comparados a indivíduos com DFP que praticavam níveis moderados de atividade física. Uma explicação para esse fato é que indivíduos que apresentam maiores níveis de atividade física são mais expostos a elevados estresses da articulação femoropatelar durante atividades de descarga de peso, o que pode levar à degradação da cartilagem articular e contribuir para o desenvolvimento e agravamento da DFP [24-26]. Portanto, o maior nível de atividade física apresentado por adolescentes do sexo masculino parece ser o responsável pela maior intensidade da DFP.

Uma vez que o nível de dor se apresenta elevado em adolescentes do sexo masculino, a repercussão desta pode ter efeito prejudicial na qualidade de vida. Os resultados do presente estudo mostram que adolescentes do sexo masculino com DFP obtiveram menores escores no domínio qualidade de vida do KOOS. Complementarmente, outros estudos reforçam este entendimento, por exemplo De Oliveira Silva et al. [3] descreveram uma relação entre o nível de DFP e qualidade de vida de adolescentes atletas, com o aumento dos sintomas sendo associado com a diminuição da qualidade de vida. Da mesma forma,
Cheung et al. [15] exploraram a relação entre a gravidade dos sintomas da DFP e o correspondente impacto na qualidade de vida, função e saúde mental de atletas profissionais e amadores. Os autores encontraram que quanto maior a gravidade dos sintomas da DFP menor a saúde mental, qualidade de vida e função de atletas com DFP. Desse modo, o presente estudo, corroborando com achados prévios, mostra que a maior intensidade dos sintomas da DFP em adolescentes do sexo masculino afeta não apenas a saúde física, mas também a qualidade de vida dos mesmos.

Devido à possível relação entre dor, nível de atividade física e qualidade de vida, muitos adolescentes cessam ou diminuem o seu nível de atividade física. Rathleff et al. [27] encontraram em um estudo longitudinal que grande parte dos adolescentes com DFP deixaram de praticar atividade física em dois anos de acompanhamento devido à dor no joelho. Entretanto, é fundamental que os adolescentes continuem a realizar atividade física, pois a sua redução pode gerar efeitos deletérios na capacidade cardiorrespiratória, causar aumento da concentração adiposa e diminuir o estado geral de saúde [28,29]. Portanto, uma alternativa para lidar com esse problema é o aumento gradual da atividade física e treinamento com aumento gradual de cargas para melhor adaptação desses adolescentes às demandas biomecânicas necessárias durante atividades físicas [30]. Além disso, o tratamento dessa população por meio de exercícios com acompanhamento de um profissional da saúde parece ser efetivo na redução dos sintomas, auxiliando-os ao retorno das atividades físicas normais $[12,30]$.

Este estudo possui algumas limitações que precisam ser reconhecidas. Primeiramente, a amostra possui apenas a população de adolescentes. Embora existam poucos estudos com adolescentes e seja importante incluí-los, os resultados desse estudo podem não ser extrapolados para a população adulta. Apesar de a DFP ser uma condição presente, na maioria das vezes, em pessoas fisicamente ativas, este estudo não incluiu um grupo de adolescentes sedentários, fato este que nos impede de observar se adolescentes sedentários com DFP também apresentam comprometimento da qualidade de vida e qual a magnitude de sua dor no joelho. Portanto, é importante que estudos futuros sejam realizados incluindo adultos do sexo feminino e masculino e indivíduos sedentários para melhor generalização dos resultados.

Em conclusão, entre adolescentes com DFP, os do sexo masculino apresentaram maiores níveis de dor no joelho e de atividade física em comparação com o sexo feminino. Além disso, os adolescentes do sexo 
masculino reportaram menor qualidade de vida do que as adolescentes do sexo feminino.

\section{NOTAS}

Apoio financeiro

Este estudo recebeu apoio da Fundação de Amparo à Pesquisa do Estado de São Paulo (FAPESP) por meio da bolsa de iniciação científica (processo número: 2015/15575-8) concedida à autora MBC.

\section{Declaração de conflito de interesses}

Os autores declaram não haver conflitos de interesses relevantes ao conteúdo deste estudo. Eles informam ter tido acesso a todos os dados obtidos e assumem completa responsabilidade pela integridade dos resultados.

\section{REFERÊNCIAS}

1. Crossley K M, Stefanik JJ, Selfe J, Collins NJ, Davis IS, Powers CM, McConnell J, Vicenzino B, Bazett-Jones DM, Esculier J-F, Morrissey D, Callaghan MJ. 2016 Patellofemoral pain consensus statement from the 4th International Patellofemoral Pain Research Retreat, Manchester. Part 1: Terminology, definitions, clinical examination, natural history, patellofemoral osteoarthritis and patient-reported outcome measures. Br J Sports Med. 2016;50(14):839-43. https:// doi.org/10.1136/bjsports-2016-096384

2. de Oliveira Silva D, Briani RV, Pazzinatto MF, Ferrari D, Aragão FA, Albuquerque CE, Alves N, Azevedo FM. Reliability and differentiation capability of dynamic and static kinematic measurements of rearfoot eversion in patellofemoral pain. Clin Biomech. 2015;30(2):144-8. https://doi.org/10.1016/j.clinbiomech.2014.12.009

3. de Oliveira Silva D, Coura MB, Waiteman M, Pradela J, Pazzinatto MF, Magalhães FH, Azevedo FM. Patellofemoral pain and sports practice: reduced symptoms and higher quality of life in adolescent athletes as compared to non-athletes. Motriz Rev Educ Fis. 2016;22(1):84-9. https://doi.org/10.1590/S1980-65742016000100011

4. Briani RV, Silva DO, Pazzinatto MF, Ferreira AS, Ferrari D, Azevedo FM. Delayed onset of electromyographic activity of the vastus medialis relative to the vastus lateralis may be related to physical activity levels in females with patellofemoral pain. J Electromyogr Kinesiol. 2016;26:137-42. https://doi.org/10.1016/j.jelekin.2015.10.012

5. Mølgaard C, Rathleff MS, Simonsen O. Patellofemoral pain syndrome and its association with hip, ankle, and foot function in 16- to 18-year-old high school students: a single-blind case-control study. J Am Podiatr Med Assoc. 2011;101(3): 215-22. https://doi.org/10.7547/1010215

6. Myer GD, Ford KR, Foss KDB, Goodman A, Ceasar A, Rauh MJ, Divine JG, Hewett TE. The incidence and potential pathomechanics of patellofemoral pain in female athletes. Clin Biomech. 2010;25(7):700-7. https://doi.org/10.1016/j. clinbiomech.2010.04.001

7. Lankhorst NE, van Middelkoop M, Crossley KM, Bierma-Zeinstra SM, Oei EH, Vicenzino B, Collins NJ. Factors that predict a poor outcome 5-8 years after the diagnosis of patellofemoral pain: a multicentre observational analysis. Br $\mathrm{J}$ Sports Med. 2015;50(14):881-6. https://doi.org/10.1136/bjsports-2015-094664

8. de Oliveira Silva D, Briani R, Pazzinatto MF, Ferrari D, Aragão F, Azevedo F. Vertical ground reaction forces are associated with pain and self-reported functional status in recreational athletes with patellofemoral pain. J Appl Biomech. 2015;31(6):409-14. https://doi.org/10.1123/jab.2015-0048

9. de Oliveira Silva D, Barton CB, Pazzinatto MF, Briani R, Azevedo F. Proximal mechanics during stair ascent are more discriminate of females with patellofemoral pain than distal mechanics. Clin Biomech. 2016;35(5):56-61. https://doi. org/10.1016/j.clinbiomech.2016.04.009

10. Rathleff MS, Vicenzino B, Middelkoop M, Graven-Nielsen T, van Linschoten R, Hölmich P, Thorborg K. Patellofemoral pain in adolescence and adulthood: same same, but different? Sport Med. 2015;45(11):1489-95. https://doi.org/10.1007/ s40279-015-0364-1

11. Rathleff CR, Baird WN, Olesen JL, Roos EM, Rasmussen S, Rathleff MS. Hip and knee strength is not affected in 12-16 year old adolescents with patellofemoral pain: a cross-sectional population-based study. PLoS One. 2013;8(11):e79153. https://doi.org/10.1371/journal.pone.0079153

12. Rathleff MS, Roos EM, Olesen JL, Rasmussen S. Exercise during school hours when added to patient education improves outcome for 2 years in adolescent patellofemoral pain: a cluster randomised trial. Br J Sports Med. 2015 Mar;49(6): 406-12. https://doi.org/10.1136/bjsports-2014-093929

13. Rathleff MS, Roos EM, Olesen JL, Rasmussen S, Arendt-Nielsen L. Lower mechanical pressure pain thresholds in female adolescents with patellofemoral pain syndrome. J Orthop Sports Phys Ther. 2013;43(6):414-21. https://doi.org/10.2519/ jospt.2013.4383

14. Rathleff CR, Olesen JL, Roos EM, Rasmussen S, Rathleff MS. Half of 12-15-year-olds with knee pain still have pain after one year. Dan Med J. 2013;60(11): A4725.

15. Cheung RT, Zhang Z, Ngai SP. Different relationships between the level of patellofemoral pain and quality of life in professional and amateur athletes. PM R. 2013;5(7):568-72. https://doi.org/10.1016/j.pmrj.2012.12.007

16. Rathleff CR, Roos EM, Olesen JL, Rasmussen S, Rathleff MS. Prevalence and severity of patellofemoral pain among adolescents: a population-based study. Proceedings of the 3rd International Patellofemoral Pain Research Retreat; 2013 Sept; Vancouver, CA. 
17. Harrison AD, Ford KR, Myer GD, Hewett TE. Sex differences in force attenuation: a clinical assessment of single-leg hop performance on a portable force plate. Br J Sports Med. 2011;45(3):198-202. https://doi.org/10.1136/bjsm.2009.061788

18. Rathleff MS, Samani A, Olesen JL, Roos EM, Rasmussen S, Christensen BH, Madeleine P. Neuromuscular activity and knee kinematics in adolescents with patellofemoral pain. Med Sci Sports Exerc. 2013;45(9):1730-9. https://doi. org/10.1249/MSS.0b013e318292be30

19. de Oliveira Silva D, Briani RV, Pazzinatto MF, Gonçalves AV, Ferrari D, Aragão FA, Azevedo FM. Q-angle static or dynamic measurements, which is the best choice for patellofemoral pain? Clin Biomech. 2015;30(10):1083-7. https:// doi.org/10.1016/j.clinbiomech.2015.09.002

20. Crossley KM, Bennell KL, Cowan SM, Green S. Analysis of outcome measures for persons with patellofemoral pain: which are reliable and valid? Arch Phys Med Rehabil. 2004;85(5):815-22. https://doi.org/10.1016/S0003-9993(03)00613-0

21. Baecke JA, Burema J, Frijters JE. A short questionanaire for the measuremnet of habitual physical activity in epidemiological studies. Am J Clin Nutr. 1982;36(5):936-42.

22. Roos EM, Roos HP, Lohmander LS, Ekdahl C, Beynnon BD. Knee Injury and Osteoarthritis Outcome Score (KOOS): development of a self-administered outcome measure. J Orthop Sports Phys Ther. 1998;28(2):88-96. https://doi.org/ 10.2519/jospt.1998.28.2.88

23. Boling M, Padua D, Marshall S, Guskiewicz K, Pyne S, Beutler A. Gender differences in the incidence and prevalence of patellofemoral pain syndrome. Scand J Med Sci Sport. 2010;20(5):725-30. https://doi.org/10.1111/j.1600-0838.2009.00996.x

24. Wallace D, Salem GJ, Salinas R, Powers CM. Patellofemoral joint kinetics while squatting with and without an external load. J Orthop Sports Phys Ther. 2002;32(4):141-48. https://doi.org/10.2519/jospt.2002.32.4.141

25. Farrokhi S, Keyak JH, Powers CM. Individuals with patellofemoral pain exhibit greater patellofemoral joint stress: a finite element analysis study. Osteoarthritis Cartilage. 2011;19(3):287-94. https://doi.org/10.1016/j.joca.2010.12.001

26. Pazzinatto MF, De Oliveira Silva D, Barton C, Rathleff MS, Briani RV, Azevedo FM. Female adults with patellofemoral pain are characterized by widespread hyperalgesia, which is not affected immediately by patellofemoral joint loading. Pain Med. 2016;17(10):1953-61. https://doi.org/10.1093/pm/pnw068

27. Rathleff MS, Rathleff CR, Olesen JL, Rasmussen S, Roos EM. Is knee pain during adolescence a self-limiting condition? Prognosis of patellofemoral pain and other types of knee pain. Am J Sports Med. 2016;44(5):1165-71. https://doi. org/10.1177/0363546515622456

28. Tammelin T, Näyhä S, Laitinen J, Rintamäki H, Järvelin MR. Physical activity and social status in adolescence as predictors of physical inactivity in adulthood. Prev Med. 2003;37(4):375-81. https://doi.org/10.1016/S0091-7435(03)001622

29. McLeod TCV, Bay RC, Parsons JT, Sauers EL, Snyder AR. Recent injury and health-related quality of Life in adolescent athletes. J Athl Train. 2009;44(6):603-10. https://doi.org/10.4085/1062-6050-44.6.603

30. Rathleff MS. Patellofemoral pain during adolescence: much more prevalent than appreciated. Br J Sports Med. 2016;50(14):831-2. https://doi.org/10.1136/bjsports-2016-096328 